\title{
Transactional Process of African American Adolescents' Family Conflict and Violent Behavior
}

\author{
Daniel Ewon Choe \\ University of Pittsburgh
}

\author{
Marc A. Zimmerman \\ University of Michigan
}

\begin{abstract}
This is the first longitudinal study of urban African American adolescents that has examined bidirectional effects between their family conflict and violent behavior across all of high school. Structured interviews were administered to 681 students each year in high school at ages 15, 16, 17, and 18 years. We used structural equation modeling to test a transactional model and found bidirectional effects between family conflict and violent behavior across the middle years of high school, while accounting for sex and socioeconomic status. Findings suggest a reciprocal process involving interpersonal conflict in African American families and adolescent engagement in youth violence.
\end{abstract}

Family conflict is a salient risk factor for African American (AA) adolescents' violent offending (Paschall, Ennett, \& Flewelling, 1996). Although reciprocated hostility in parent-child relationships exacerbates behavioral problems (Laursen \& Collins, 2009), researchers who examine family risk factors for violent offending often do not test for bidirectional influences. This longitudinal study tested a transactional model of AA adolescents' family conflict and violent behavior across all of high school. Elucidating their complex interplay can further understanding of coercive family processes and adolescent engagement in youth violence.

A family environment with low levels of conflict and high cohesion is predictive of less aggressive behavior in childhood and adolescence (Andreas \& Watson, 2009). Yet many risk factors for youth violence extend beyond the family. Community-level risks of violent behavior correlate with severe economic disadvantage, such as living in a crowded urban area and poor-quality housing (Chung \& Steinberg, 2006; Dodge \& Pettit, 2003; Loeber \& Farrington, 2000). Sociodemographic risks disproportionately affect AA adolescents and may explain their higher levels of violence compared with White youths (Paschall et al., 1996). High levels of community violence, crime, and other correlates of poverty amplify psychosocial stressors in peer and family systems and in turn contribute to violent

\footnotetext{
This research was funded by the National Institute on Drug Abuse, Grant No. DA07484 awarded to Dr. Marc Zimmerman. The research reported here does not necessarily reflect the views or policies of the National Institute on Drug Abuse.

Requests for reprints should be sent to Daniel Ewon Choe, Department of Psychology, University of Pittsburgh, 210 S. Bouquet Street, 4423 Sennott Square, Pittsburgh, PA 15260.

E-mail: dec60@pitt.edu
}

offending during adolescence (Dodge \& Pettit, 2003).

Deviant peer influences in adolescence are among the most potent risk factors for antisocial behavior (Dishion, Piehler, \& Myers, 2008), but parenting factors, such as monitoring and involvement in adolescents' lives, also critically influence adolescent adjustment (Farrington, 2009). Deviant peer influences, however, are less likely to have an enduring influence on adolescents unless exposure is stable and frequent (Bronfenbrenner \& Morris, 2006; Dodge \& Pettit, 2003). Family members are more consistently present in adolescents' lives, suggesting that family factors trump peer influences. We acknowledge substantial contributions of the broader social context to adolescent development, but this study of AA adolescents from an impoverished U.S. city focused on the interplay of their violent behavior and family conflict using a transactional framework.

The transactional perspective of developmental psychopathology emphasizes reciprocal exchanges between the individual and social context contributing to adjustment problems (Cicchetti \& Toth, 1997). This perspective originated from transactional models emphasizing the continuous interplay between parents and children in contributing to social-emotional development and caregiving behaviors (Sameroff \& Chandler, 1975). Critical to these perspectives is an analytic focus on bidirectional effects between individual and social-contextual variables typically examined in autoregressive cross-lagged models (Sameroff, 2000). Examining

(C) 2013 The Authors

Journal of Research on Adolescence (C) 2013 Society for Research on Adolescence DOI: $10.1111 /$ jora.12056 
bidirectional effects between violent behavior and family conflict in a transactional model can reveal how the two affect one another as a reciprocal process of parent-child hostility.

Family relationships, particularly with parents, undergo substantive transformations in early adolescence, but remain the most powerful influences on social-emotional development (Laursen \& Collins, 2009). During adolescence, youth gradually spend less time with family as they strive for autonomy, which often exacerbates family conflict as parents and adolescents negotiate the increasing independence youth desire (Collins \& Steinberg, 2006). The frequency of parent-child conflict decreases from early to late adolescence, but its emotional intensity increases from early to middle adolescence and then stabilizes (Laursen, Coy, \& Collins, 1998). Contrary to popular belief, consistent high-intensity fighting among family is not normative during adolescence, but it is characterized by more hostile and angry disputes than interpersonal conflict with family at other ages (Collins \& Steinberg, 2006). Thus, frequency of parent-child conflict decreases after peaking in early adolescence, while its emotional intensity increases to a stable level in middle adolescence; yet, we do not know how adolescent violence influences this.

Cross-sectional evidence indicates that more family conflict is associated with more violent behavior among urban AA youth aged 11-19 years (DuRant, Cadenhead, Pendergrast, Slavens, \& Linder, 1994). Moreover, 7th- and 8th-grade AA males reported more family conflict than White males, which was associated with their greater violent behavior (Paschall et al., 1996). These findings suggest that elevated levels of AA adolescents' violent behavior may be due to an increased risk of family conflict, but they do not clarify whether there is a reciprocal association or whether it changes over time. Longitudinal study of the bidirectional interplay of violent behavior and family conflict can elucidate a dynamic process within AA families that may contribute to more interpersonal conflict and youth violence.

Cui, Donnellan, and Conger (2007) discovered that more marital conflict over child rearing when adolescents were 12-14 years old predicted more delinquent behavior a year later. More delinquency when adolescents were 13-15 years old in turn predicted more marital conflict the next year. In another study, marital conflict exacerbated children's behavioral problems, which consequently worsened marital conflict (Jenkins, Simpson, Dunn,
Rasbash, \& O'Connor, 2005). These findings suggest a bidirectional association between adolescents' antisocial behavior and family conflict in which they reinforce one another. But few researchers have examined reciprocal influences in AA families over periods longer than a year or two.

This is the first study to examine the transactional interplay of urban AA adolescents' violent behavior and family conflict across all 4 years of high school. We controlled for socioeconomic status (SES) and sex in analyses, because sociodemographic factors are some of the most consistent and robust predictors of antisocial behavior in adolescence (Chung \& Steinberg, 2006; Dodge \& Pettit, 2003; Farrington, 2009). Male adolescents have reported more violent behavior than females (DuRant et al., 1994), and female adolescents have reported greater family conflict (Daigle, Cullen, \& Wright, 2007). We hypothesized that AA adolescents' family conflict and violent behavior would predict higher levels of one another during high school through bidirectional effects, as supported by prior research (Cui et al., 2007; Jenkins et al., 2005).

\section{METHOD}

\section{Participants}

This study included 681 AA adolescents (49\% male) selected from an urban city with a homicide rate over twice the national average (Michigan Department of Community Health, 2006). Initial recruitment selected 979 students (80\% AA) for a larger study of school dropout and substance use (Zimmerman \& Schmeelk-Cone, 2003). Students enrolled in 9th grade in four public high schools with a grade point average of 3.0 or below were eligible for participation. Students diagnosed as emotionally impaired or developmentally disabled were not eligible. Participants were almost 15 years old $(M=14.86)$ in 9 th grade. During this time, $39 \%$ of adolescents lived with their mothers and siblings, 25\% lived with both parents and extended relatives, $10 \%$ lived with mothers and extended relatives, $10 \%$ lived with mothers and stepfathers, and the remaining lived with extended relatives or did not respond. In 10th grade, $34 \%$ of adolescents who responded reported a change in household structure: 39\% lived with mothers and siblings, $35 \%$ lived with both parents, $10 \%$ lived with mothers and extended relatives, and the remaining lived with extended relatives or did not respond. In 11th and 12th grade, $37 \%$ and $29 \%$ of adolescents 
reported household changes, although no other structural data were provided.

\section{Procedure}

Trained interviewers conducted structured hourlong interviews with participants during school hours annually at each grade in high school. Participants who dropped out of school were interviewed in their homes or a community setting. School dropout was determined at age 20; 115 participants $(17 \%)$ reported that they did not complete high school or receive a GED. Participants were paid for participating and informed that all information would be confidential.

\section{Measures}

Violent behavior. Seven items assessed participants' fighting inside and outside of school or work, fighting in a group, hitting a teacher or supervisor, using or carrying a knife, razor, or gun, and whether they ever hurt someone badly enough to need medical aid. Participants indicated how often they engaged in each behavior during the past 12 months using a 5-point response scale $(1=0$ times; $5=4$ or more times). Six of seven items were aggregated into two 3-item parcels corresponding with fighting (mean $\alpha=.51$ ) and weapon use (mean $\alpha=.62$ ). Latent variables for violent behavior at each grade were created with these two parcels and the single item "hurt someone badly enough to need medical aid."

Family conflict. Five items assessed participants' family members fighting a lot, losing their tempers, throwing things when angry, hitting, and criticizing each other. Participants selected answers best representing their family situation during the last 12 months using a 4-point response scale $(1=$ hardly ever; $4=$ often). The "losing their tempers" and "criticizing each other" items were aggregated into a parcel indicating family social-emotional stress (mean $\alpha=.67$ ), whereas the "throwing things when angry" and "hitting" items were aggregated into a parcel for family physical fighting (mean $\alpha=.70$ ). Latent variables for family conflict at each grade were created with these two parcels and the single item "family members fight a lot."

Socioeconomic status. SES was assessed by prestige scores of parents' occupation (Nakao \& Treas, 1990). When both parents reported, the higher of the two scores was used. The mean prestige score for the sample was $39.81(S D=10.48)$, indicating a blue-collar occupation.

\section{Data Analysis Plan}

Preliminary analysis of sample attrition across high school was conducted with SPSS 19 and only a sex differences was found. More males $(n=48)$ than females $(n=29)$ were in the attrition group $(11.3 \%), \chi^{2}(1)=6.00, p=.014$. We conducted structural equation modeling (SEM) using Mplus 6.1 with maximum likelihood (Muthén \& Muthén, 2010) to construct latent variables for violent behavior and family conflict and a transactional model. We evaluated models based on chi-square $\left(\chi^{2}\right)$, comparative fit index (CFI), estimated rootmean-square error of approximation (RMSEA) and its $90 \%$ confidence interval. RMSEA values $\leq .05$ indicate close approximate fit. CFI values $>.90$ reflect reasonably good fit.

\section{RESULTS}

Latent variables for violent behavior and family conflict were created with parcels and individual items. Parcels representing fighting (mean $\beta=.76$, $p s<.001$ ) and weapon use (mean $\beta=.70$, $p \mathrm{~s}<.001)$ and the item, "hurt someone badly enough to need medical aid" (mean $\beta=.74$, $p s<.001)$ were significant indicators of violent behavior. Parcels representing family social-emotional stress (mean $\beta=.77, p s<.001$ ) and physical fighting (mean $\beta=.72, p s<.001$ ) and the item, "family members fight a $\operatorname{lot}^{\prime}$ (mean $\beta=.67$, $p s<.001)$ were significant indicators of family conflict. Factor loadings indicated that items adequately fit latent factors (see Table 1). An autoregressive cross-lagged model was specified to examine bidirectional effects between AA adolescents' family conflict and violent behavior across 4 years of high school (see Figure 1). Covariances between error terms of concurrent measures estimated shared variance in measurement error. Sex and SES were included as covariates, but are not shown in Figure 1.

The model in Figure 1 produced excellent fit indices. The model explained $40 \%$ of the variance in 12th-grade family conflict, $39 \%$ in 11th-grade family conflict, $44 \%$ in 10th-grade family conflict, $3 \%$ in 9th-grade family conflict, $55 \%$ in 12th-grade violent behavior, $52 \%$ in 11th-grade violent behavior, $39 \%$ in 10th-grade violent behavior, and $2 \%$ in 9th-grade violent behavior. Standardized parameter estimates in Figure 1 indicate significant lagged 
TABLE 1

Unstandardized and Standardized Measurement Model Estimates for Figure 1 (Standard Errors)

\begin{tabular}{|c|c|c|c|}
\hline Parameter estimate & Unstandardized & Standardized & $p$ \\
\hline 12th Violent behavior $\rightarrow$ fighting & 1.00 & .79 & - \\
\hline 12th Violent behavior $\rightarrow$ weapon use & $0.74(.04)$ & .74 & .000 \\
\hline 12th Violent behavior $\rightarrow$ hurt someone badly & $0.27(.02)$ & .71 & .000 \\
\hline 12th Family conflict $\rightarrow$ family S.E. stress & 1.00 & .81 & - \\
\hline 12th Family conflict $\rightarrow$ family physical fighting & $0.55(.04)$ & .68 & .000 \\
\hline 12th Family conflict $\rightarrow$ family fights a lot & $0.44(.03)$ & .74 & .000 \\
\hline 11th Violent behavior $\rightarrow$ fighting & 1.00 & .80 & - \\
\hline 11th Violent behavior $\rightarrow$ weapon use & $0.79(.05)$ & .71 & .000 \\
\hline 11th Violent behavior $\rightarrow$ hurt someone badly & $0.30(.02)$ & .78 & .000 \\
\hline 11th Family conflict $\rightarrow$ family S.E. stress & 1.00 & .77 & - \\
\hline 11th Family conflict $\rightarrow$ family physical fighting & $0.63(.04)$ & .77 & .000 \\
\hline 11th Family conflict $\rightarrow$ family fights a lot & $0.38(.03)$ & .64 & .000 \\
\hline 10th Violent behavior $\rightarrow$ fighting & 1.00 & .75 & - \\
\hline 10th Violent behavior $\rightarrow$ weapon use & $0.66(.04)$ & .68 & .000 \\
\hline 10th Violent behavior $\rightarrow$ hurt someone badly & $0.30(.02)$ & .74 & .000 \\
\hline 10th Family conflict $\rightarrow$ family S.E. stress & 1.00 & .73 & - \\
\hline 10th Family conflict $\rightarrow$ family physical fighting & $0.72(.05)$ & .72 & .000 \\
\hline 10th Family conflict $\rightarrow$ family fights a lot & $0.45(.03)$ & .66 & .000 \\
\hline 9th Violent behavior $\rightarrow$ fighting & 1.00 & .70 & - \\
\hline 9th Violent behavior $\rightarrow$ weapon use & $0.96(.07)$ & .66 & .000 \\
\hline 9th Violent behavior $\rightarrow$ hurt someone badly & $0.51(.04)$ & .72 & .000 \\
\hline 9th Family conflict $\rightarrow$ family S.E. stress & 1.00 & .75 & - \\
\hline 9th Family conflict $\rightarrow$ family physical fighting & $0.76(.05)$ & .73 & .000 \\
\hline 9th Family conflict $\rightarrow$ family fights a lot & $0.43(.03)$ & .63 & .000 \\
\hline
\end{tabular}

Note. Numbers before variables indicate grades when measured. Covariances of latent variable indicators estimated but not shown. S.E. $=$ Social-Emotional.

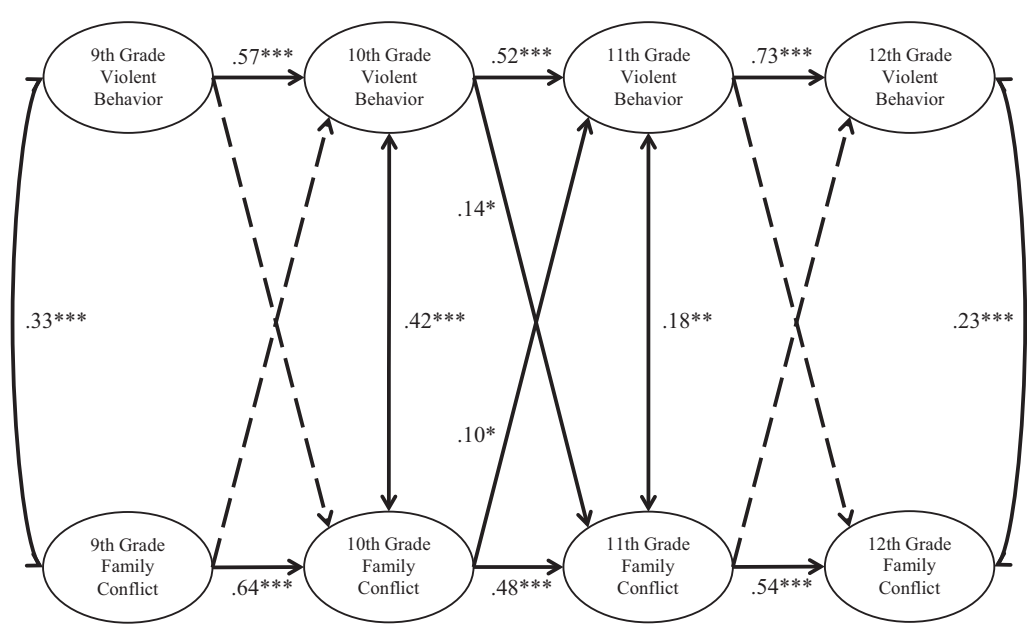

FIGURE 1 Transactional model of African American adolescents' family conflict and violent behavior across high school (standardized solution, $N=681) \cdot \chi^{2}(229)=404.18, p<.001$; CFI $=.97$; RMSEA $=.03,90 \%$ CI [.03, .04]. Error and disturbance terms and sex and SES covariates are not shown.

$* p<.05 ; * * p<.01 ; * * * p<.001$.

effects of family conflict and violent behavior, suggesting construct stability through high school.

We hypothesized that urban AA adolescents' family conflict and violent behavior would predict higher levels of one another during high school through bidirectional effects. As shown in Figure 1, 10th-grade family conflict predicted higher levels of 11th-grade violent behavior $(\beta=.10, p<.05)$ and 10th-grade violent behavior predicted higher levels of 11th-grade family conflict $(\beta=.14$, $p<.05)$. Bidirectional effects were found while accounting for sex and SES. Being male was 
associated with more violent behavior from 9th to 11th grade. Being female was associated with higher levels of family conflict in 9th and 12th grade.

\section{DISCUSSION}

This is the first study to test a transactional model of AA adolescents' violent behavior and family conflict across all 4 years of high school. We sought to elucidate bidirectional effects in AA families from an urban U.S. city characterized by high levels of violence, crime, and poverty. Modest bidirectional effects across the middle years of high school indicated that heightened levels of family conflict and violent behavior exacerbated one another from 10 th to 11th grade. These findings suggest a reciprocal process of parent-child hostility involving interpersonal conflict in AA families and adolescent engagement in youth violence.

In accord with our hypothesis and previous research (DuRant et al., 1994; Paschall et al., 1996), high levels of family conflict in 10th grade predicted more violent behavior in 11th grade. This is consistent with the literature indicating that a stressful family environment contributes to more stable violent aggression during adolescence (Andreas \& Watson, 2009; Dodge \& Pettit, 2003; Farrington, 2009). We further discovered that AA adolescents' violent behavior in 10th grade predicted higher levels of family conflict over the next school year. Complementing literature showing that family conflict is a risk factor for youth violence (Paschall et al., 1996), adolescents' violent behavior also increased risk of interpersonal conflict in AA families.

Evidence of bidirectional effects between family conflict and violent behavior can help delineate reciprocal family interactions and how they may contribute to youth violence. Researchers have found bidirectional effects between children and adolescents' behavioral problems and marital conflict over a shorter period of time than in our study (Cui et al., 2007; Jenkins et al., 2005). These researchers proposed that family conflict and youth behavioral problems operate as stressors on the family environment, thereby exacerbating familywide levels of adjustment problems and interpersonal conflict. In this study, AA adolescents' violent behavior peaked in 10th grade and predicted higher levels of family conflict in 11th grade, suggesting that family members responded to adolescents' increasingly violent behavior with more arguing and fighting. Family systems were disrupted by adolescents' worsening violence, while family conflict exacerbated adolescents' violent behavior across the middle years of high school.

Adolescents experience numerous consequences of their violent behavior, such as altercations with police and physical and psychological injury. These experiences also affect family members who often become frustrated and angry with adolescents and respond in hostile ways. For example, legal sanctions mediate effects of adolescent delinquency on poor parenting, such that parents respond to adolescents' arrests with negative parenting behaviors (Stewart, Simons, Conger, \& Scaramella, 2002). An adolescent's perpetration of violence can lead to conflict with family in many ways. In worst cases, adolescent violence may be manifested in the context of abusive family conflict and parent-child hostility. Not only does family violence contribute to violent behavior, it disproportionately affects AA families (Paschall et al., 1996).

Family socializing influences are critical in managing adolescent adjustment, but reciprocated hostility jeopardizes this by disrupting family processes (Collins \& Steinberg, 2006; Laursen \& Collins, 2009). Researchers have suggested adolescent violence is functional in deflecting aversive behavior from family members and eliciting their attention-a means for adolescents to maintain a sense of control in a dysfunctional home environment (Snyder \& Patterson, 1995). Although bidirectional effects of AA adolescents' family conflict and violent behavior in this study were modest, they enhance our understanding of reciprocal processes affecting the family environment and adolescent development. Youth who report more parental communication, monitoring, and support cultivate better interpersonal relationships and develop fewer antisocial problems (Dodge \& Pettit, 2003; Laursen \& Collins, 2009). In AA families where these social resources are scarce and conflict is intense, adolescents may find an escape in their peers. The combination of a hostile family environment and more time spent in the community with peers may offer AA adolescents plentiful opportunities to engage in violence.

Several limitations of this study warrant caution when interpreting the results. Differences in household structure were not controlled for due to frequent mobility within households and the difficulty of controlling for their changes. Parental separation is a risk factor for conduct problems; yet, adolescents from broken homes do not differ in delinquent behavior from their peers who have high-conflict families (Dodge \& Pettit, 2003; Farrington, 2009). This suggests household structure is as powerful a predictor of antisocial behavior as the 
interpersonal conflict that constitutes it. Research that distinguishes between family structures or exclusively samples families of a certain composition is needed to account for household configuration.

Our investigation focused on family conflict and adolescent violence, but, as repeatedly mentioned, deviant peer influences play a potent role in antisocial behavior (Dishion et al., 2008). Models of youth violence that focus on one social-contextual system are criticized as being overly simplistic, as parental and peer influences operate in tandem to contribute to adolescents' antisocial behavior (Chung \& Steinberg, 2006; Dodge \& Pettit, 2003). Peer influences and adolescent delinquency likely operate in a reciprocal fashion where adolescents select and are selected by deviant peer groups that escalate their antisocial behavior. Future research that assesses concomitant process variables from family and peer microsystems may further elucidate transactional processes that reinforce violent behavior during adolescence.

Lastly, findings from this study are limited in generalizability to mostly low-income AA adolescents raised in an urban city characterized by high levels of violence and crime. AA adolescents were at greater risk of violent offending than the general population, and therefore more likely to leave the study due to reasons such as juvenile detention. Bidirectional effects were relatively modest in size, but powerful enough to emerge without these extreme cases.

Our study contributed the first evidence of bidirectional associations between AA adolescents' family conflict and violent behavior across the middle years of high school. A transactional perspective examining adolescent development within an evolving social context is needed to enhance the relative dearth of literature on adolescent-family transactions. Reciprocal family processes can stress adolescents' interpersonal relations and jeopardize their adaptation across important developmental transitions that represent both windows of vulnerability to stress and foci for preventive interventions. Replication of these findings, a focus on mechanisms that mediate effects in both directions, and integration of process variables across ecological systems can inform interventions and reduce costs associated with youth violence.

\section{REFERENCES}

Andreas, J. B., \& Watson, M. W. (2009). Moderating effects of family environment on the association between children's aggressive beliefs and their aggression trajectories from childhood to adolescence. Development and Psychopathology, 21, 189-205.

Bronfenbrenner, U., \& Morris, P. A. (2006). The bioecological model of human development. In W. Damon (Series Ed.) \& R. M. Lerner (Volume Ed.), Handbook of child psychology: Theoretical models of human development (Vol. 1, 6th ed., pp. 793-828). New York, NY: Wiley.

Chung, H. L., \& Steinberg, L. (2006). Relations between neighborhood factors, parenting behaviors, peer deviance, and delinquency among serious juvenile offenders. Developmental Psychology, 42, 319-331.

Cicchetti, D., \& Toth, S. L. (1997). Transactional ecological systems in developmental psychopathology. In S. S. Luthar, J. Burack, D. Cicchetti, \& J. Weisz (Eds.), Developmental psychopathology: Perspectives on adjustment, risk, and disorder (pp. 317-349). New York, NY: Cambridge University Press.

Collins, W. A., \& Steinberg, L. (2006). Adolescent development in interpersonal context. In W. Damon \& R. M. Lerner (Series Eds.) \& N. Eisenberg (Vol. Ed.), Handbook of child psychology: Social, emotional, and personality development (Vol. 3, 6th ed., pp. 1003-1067). Hoboken, NJ: John Wiley \& Sons.

Cui, M., Donnellan, M. B., \& Conger, R. D. (2007). Reciprocal influences between parents' marital problems and adolescent internalizing and externalizing behavior. Developmental Psychology, 43, 1544-1552.

Daigle, L. E., Cullen, F. T., \& Wright, J. P. (2007). Gender differences in the predictors of juvenile delinquency: Assessing the generality-specificity debate. Youth Violence and Juvenile Justice, 5, 254-286.

Dishion, T. J., Piehler, T. F., \& Myers, M. W. (2008). Dynamics and ecology of adolescent peer influence. In M. J. Prinstein \& K. A. Dodge (Eds.), Understanding peer influence in children and adolescents (pp. 72-93). New York, NY: Guilford Press.

Dodge, K. A., \& Pettit, G. S. (2003). A biopsychosocial model of the development of chronic conduct problems in adolescence. Developmental Psychology, 39, 349-371.

DuRant, R. H., Cadenhead, C., Pendergrast, R. A., Slavens, G., \& Linder, C. W. (1994). Factors associated with the use of violence among urban black adolescents. American Journal of Public Health, 84, 612-617.

Farrington, D. P. (2009). Conduct disorder, aggression, and delinquency. In R. M. Lerner \& L. Steinberg (Eds.), Handbook of adolescent psychology: Individual bases of adolescent development (Vol. 1, 3rd ed., pp. 683-722). Hoboken, NJ: John Wiley \& Sons.

Jenkins, J., Simpson, A., Dunn, J., Rasbash, J., \& O'Connor, T. G. (2005). Mutual influence of marital conflict and children's behavior problems: Shared and nonshared family risks. Child Development, 76, 24-39.

Laursen, B., \& Collins, W. A. (2009). Parent-child relationships during adolescence. In R. M. Lerner \& L. Steinberg (Eds.), Handbook of adolescent psychology: Contextual influences on adolescent development (Vol. 2, 3rd ed., pp. 3-42). New York, NY: Wiley. 
Laursen, B., Coy, K. C., \& Collins, W. A. (1998). Reconsidering changes in parent-child conflict across adolescence: A meta-analysis. Child Development, 69, 817-832.

Loeber, R., \& Farrington, D. P. (2000). Young children who commit crime: Epidemiology, developmental origins, risk factors, early interventions, and policy implications. Development and Psychopathology, 12, 737-762.

Michigan Department of Community Health. (2006). 1989-2004 Michigan resident death files. Lansing, MI: Vital Records and Health Data Development Section, $\mathrm{MDCH}$.

Muthén, L. K., \& Muthén, B. O. (2010). Mplus version 6.1 user's guide. Los Angeles, CA: Muthén \& Muthén.

Nakao, K., \& Treas, J. (1990). Computing 1989 occupational prestige scores (GSS Methodological Report No. 70). Chicago, IL: National Opinion Research Center.

Paschall, M. J., Ennett, S. T., \& Flewelling, R. L. (1996). Relationships among family characteristics and violent behavior by Black and White male adolescents. Journal of Youth and Adolescence, 25, 177-197.
Sameroff, A. J. (2000). Developmental systems and psychopathology. Development and Psychopathology, 12, 297-312.

Sameroff, A. J., \& Chandler, M. (1975). Reproductive risk and the continuum of caretaking casualty. In F. D. Horowitz (Ed.), Review of child development research (4th ed., pp. 187-244). Chicago, IL: University of Chicago Press.

Snyder, J., \& Patterson, G. R. (1995). Individual differences in social aggression: A test of a reinforcement model of socialization in the natural environment. Behavior Therapy, 26, 371-391.

Stewart, E. A., Simons, R. L., Conger, R. D., \& Scaramella, L. V. (2002). Beyond the interactional relationship between delinquency and parenting practices: The contribution of legal sanctions. Journal of Research in Crime and Delinquency, 39, 36-59.

Zimmerman, M. A., \& Schmeelk-Cone, K. H. (2003). A longitudinal analysis of adolescent substance use and school motivation in African American youth. Journal of Research on Adolescence, 13, 185-210. 